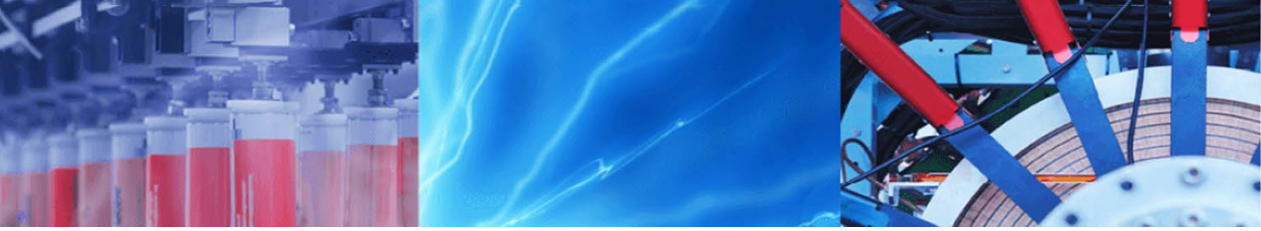

Research Article

\title{
Using deep reinforcement learning approach for solving the multiple sequence alignment problem
}

\author{
Reza Jafari $^{1} \cdot$ Mohammad Masoud Javidi $^{1}$. $\cdot$ Marjan Kuchaki Rafsanjani ${ }^{1}$
}

(c) Springer Nature Switzerland AG 2019

\begin{abstract}
In the present paper, we use a deep reinforcement learning (DRL) approach for solving the multiple sequence alignment problem which is an NP-complete problem. Multiple Sequence Alignment problem simply refers to the process of arranging initial sequences of DNA, RNA or proteins in order to maximize their regions of similarity. Multiple Sequence Alignment is the first step in solving many bioinformatics problems such as constructing phylogenetic trees. In this study, our proposed approach models the Multiple Sequence Alignment problem as a DRL problem and utilizes long short-term memory networks for estimation phase in the reinforcement learning algorithm. Furthermore, the actor-critic algorithm with experience-replay method is used for much quicker convergence process. Using deep Q-learning (an RL approach) and Q-network overcomes the complexity of other approaches. The experimental evaluation is performed on 8 different real-life datasets and in every used dataset our approach outperforms other well-known approaches and tools such as MAFFT, ClustalW, and other heuristic approaches in case of scoring in solving the MSA problem.
\end{abstract}

Keywords Bioinformatics · Multiple sequence alignment · Deep reinforcement learning $\cdot$ LSTM

\section{Introduction}

Multiple Sequence Alignment (MSA) problem [1] is one of the most important problems in bioinformatics. It is the first step toward any bioinformatics study of protein, Ribonucleic acid or Deoxyribonucleic acid. Simply, it refers to the process of positioning primary sequences of DNA, RNA or protein in order to identify their regions of similarity (propose a permutation of the sequences).

MSA is an NP-complete problem [2] therefore, many heuristic approaches and algorithms have been applied toward MSA problem, in order to reach an optimal alignment such as genetic algorithm with using custom operations [3-5], hidden Markov model [6], fuzzy logic [7] reinforcement learning $[8,9]$ and many other tools $[10,11]$.

An MSA can be useful for many scientific targets such as inferring the presence of ancestral relationships between the mentioned sequences. The MSA main applications in bioinformatics are extrapolation (for example assigning an uncharacterized sequence to a protein family), phylogenetic analysis, pattern identification and DNA regulatory elements.

Finding regions of similarity in DNA, protein, RNA sequences provides information such as structural, functional and evolutionary relationships between mentioned sequences. The importance of MSA shows itself in phylogenetic trees topic, in which a phylogenetic tree cannot be constructed without aligning its consisting sequences.

In this paper, we apply the deep reinforcement learning (DRL) [12] approach for the MSA problem. This approach makes the learning process much faster by using neural networks (Q-networks) as approximators in estimation part in the RL algorithm. We benefit our model by using long-short-term memory (LSTM) networks due to their ability to memorize long dependencies and the sequential character of the MSA problem. Also, we use the critic-actor

Mohammad Masoud Javidi, javidi@uk.ac.ir; Reza Jafari, r.jafari@math.uk.ac.ir; Marjan Kuchaki Rafsanjani, kuchaki@uk.ac.ir| ${ }^{1}$ Computer Science Department, Shahid Bahonar University of Kerman, Kerman, Iran.

SN Applied Sciences (2019) 1:592 | https://doi.org/10.1007/s42452-019-0611-4

Received: 20 March 2019 / Accepted: 14 May 2019 / Published online: 18 May 2019 
algorithm with an experience replay mechanism which results in more efficient algorithm performance. The reason and the motivation of using neural networks and DRL is this point that, to our knowledge DRL approach and neural networks especially LSTMs networks has not been used in the literature to address MSA problem so far and due to the decision making and NP-complete character of the MSA problem, in which we only can estimate the solution, the $\mathrm{RL}$ algorithm is a right choice and also, this fact that neural networks are great universal approximators. Choosing these structure and approach leads us to a reasonable result.

The rest of the paper is organized as follow: in Sect. 2, the MSA problem and its main aspects are mentioned. Sect. 3 contains the related works that proposed toward MSA problem. Section 4 presents the basic definitions and fundamentals of deep reinforcement learning and LSTMs. In Sect. 5 we introduce our approach toward MSA problem. Comparisons between DRL approach and related works from the literature are given in Sect. 6 and finally, Sect.7 outlines some conclusions and expresses future developments.

\section{Multiple sequence alignment and its characteristics}

Finding similar regions in DNA, RNA, protein sequences are crucial in many bioinformatics problems such as predicting the secondary structure of proteins, evolutionary origins of the chosen sequences, finding mutations which occurred in proteins or DNA and etc. Finding the maximum number of common regions in sequences is MSA problem's goal and it can be fulfilled by placing the sequences in an optimal order.

Many cost measures introduced for MSA problem such as SP score [13], PAM and BLOSUM. However, the SP score used much more in literature therefore, we use SP (sum of pairs) score as well. This score sums the all possible pairwise combinations of sequences characters over all of the columns.

$\mathrm{SP}=\sum_{i=1}^{p} \sum_{j=1}^{n-1} \sum_{k=j+1}^{n} \operatorname{score}\left(\operatorname{col}_{i}^{j}, \operatorname{col}_{i}^{k}\right)$

where $P$ is the number of columns; $n$ is the number of sequences; $\mathrm{Col}_{i}$ shows the column i; $\mathrm{Col}_{i}$ shows the jth character from ith column and score $\left(\operatorname{col}_{i}^{j}\right.$, coll $\left._{i}^{k}\right)$ represents the comparing score between mentioned characters [13].

Following example defines the basic terms in MSA problem. Consider sequence (1) and (2) in Fig. 1. three possibilities could happen in the process of comparing two

\section{s1: A T C G$$
\text { s2: - T C A }
$$

Fig. 1 Two sequence of nucleotides

characters in one column. $A$ is the first character in the first sequence and gap (-) is the first character in the sequence 2 when this situation occurs between any character with a gap character a penalty value $(-2)$ assigns to this occurrence. In the second column both of the characters are $T$, this occurrence called a match and its reward value is +2 . In the third column, we also have a match and in the last column there is a mismatch occurrence and its penalty value is -1 . This process is a conventional value assignment between most of the proposed approaches.

As mentioned earlier, the MSA problem addressed by many computational approaches. Three main categories are dynamic programming approach, progressive alignment method, and iterative refinement method. Dynamic programming approach works fine for pairwise alignment but using this approach for the MSA problem is very timewise expensive (cost of the computations grows exponentially). In progressive alignment two sequences are chosen and aligned by standard pairwise alignment then another sequence aligned with the previously aligned sequences and the process carries on until the end. Iterative refinement method works similarly to progressive alignment method but in the iterative refinement method, once our new sequence is added to the alignment, the initially aligned sequences are repeatedly realigned in order to provide the best alignment.

In the following section, a few related works will be discussed.

\section{Related works}

As mentioned earlier, the MSA problem is an NP-complete problem. Therefore, many heuristic approaches proposed for solving this particular important bioinformatic problem.

Chen and et al. [14] applied the Ant Colony Optimization (ACO) algorithm to the MSA problem. It was a partitioning based approach which had three phases. This approach was useful in large-scale benchmark instances. In their paper, it was claimed that they improved the solution time and alignment's quality by using the locality structure of the problem. Their approach contains three stages for dividing the set of sequences into some subsections. They used an automated and sub-optimal partitioning procedure and then they performed the ant 
colony-based alignment on each sub-section and finally at the last stage by assembling the result of each subsection they reached the alignment of the original sequences. The usage of the ant colony algorithm avoids the local optimal traps.

Rasmussen and et al. [6] Combined hidden Markov models with a particle swarm optimization for solving MSA problem. They conquered hidden Markov models' hard computational character by using particle swarm optimization with evolutionary algorithms to train hidden Markov models. Their approach yielded better results for protein sequences datasets than other hidden Markov models such as Baum-welch and simulated annealing. Their approach was able to increase both the quality of alignments and the long-odds score of the hidden Markov models.

Many genetic-based approaches have been proposed for the MSA problem so far. First ones who used genetic algorithm were Chen and Lin [3]. Their approach provides the best cut points with divide and conquers techniques. In this approach, a genetic algorithm was used for finding the best cut-points and then each subset of the sequences aligned by the dynamic programming techniques. They claimed that their approach reduces the space complexity and increases the match columns and scores. Another use of the genetic algorithm toward the MSA problem proposed by Agarwal [4], in which custom operations such as gap shuffles mutation introduced. In fact, producing offsprings of improved fitness resulted in reaching better alignments.

The first use of the RL algorithm for solving the MSA problem proposed by Mircea and et al. [8,9]. They also proposed an approach which was the results of combining the Q-learning algorithm with two variations of a sequence alignment algorithm and three distinct action selectors. They also utilized an intelligent look-ahead action selection method and an affine gap penalization process which increases scores and decreases the interior gaps. The main strength of their proposed approach was the low computational time.

Nasser and et al. [7] proposed an approach with the use of fuzzy logic. The reason for using fuzzy logic was the fact that fuzzy logic has more tolerance to the errors in subsequences' miss matching and matching. They used fuzzy logic for estimate matching of sub-sequences. They also built a fuzzy assembler in order to deal with low-quality data.

Rubio-Largo and et al. [15] used Hybrid multi-objective artificial bee colony toward the MSA problem. They considered two objective functions for maintaining the consistency and the quality of the alignments, the number of totally conserved columns score and weighted sum-of-pairs function. The main drawback of their approach was its long run-time than other existing methods.

Lalwani and et al. [16] introduced An Efficient Bi-Level Discrete particle swarm optimization Variant for the MSA problem. Their approach consists of two levels. The first level dedicated to optimizing dimension for the complete swarm and second level is responsible for optimizing each particle's position. Their approach addressed the discrete problem with exponentially decreasing inertia weight strategy.

Two of the most well-known tools for solving the MSA problem are ClustalW [10] and MAFFT [11]. They are matrix-based tools. They also have a quick algorithm and also reasonable processing time for large datasets. ClustalW uses progressive alignment method. It finds an optimal alignment by aligning the most similar sequences and carries on with the least similar ones. It has a matrix-based algorithm and its process steps are: first, it computes a rough distance matrix between each pair of mentioned sequences and in its next step, a neighbor-joining method is used for creating an overall guide tree with midpoint rooting. This tool time complexity is $O\left(\mathrm{~N}^{2}\right)$. ClustalW usually performs better in datasets which consists of sequences with a high degree of divergence. In [17], chenna and et al. explained and introduced other ClustalW versions and their frameworks and they also introduced new features include NEXUS and FASTA format output, printing range numbers and faster tree calculation.

Multi Alignment using Fourier Transform (MAFFT) also is a matrix-based tool and uses progressive alignment method. In its process, the sequences are clustered with fast Fourier transform [5]. MAFFT tool steps for finding an optimal alignment are: constructing a rough distance matrix by counting the number of shared tuples (between every sequence pair), building a guide tree and finally based on the branching order an alignment will be reached.

Another method which used for solving the MSA problem was the ensemble learning method. In [18], Katoh and et al. proposed an approach which was capable of producing an alignment even when the unaligned sequences are short and fragmentary.

AlexSys [19] is a knowledge-based expert system for multi-sequence alignment analysis and construction. AlexSys used tree-decision method. Decision trees are used in may machine learning and computer vision problems $[20,21]$. As the name goes it uses a tree-like structure of decisions. Particularly in decision analysis it is used for determining the expected values of competing alternatives [22, 23]. AlexSys determines a decisiontree approach to predict whether given sequences are 
weakly or strongly aligned with each method (classification problem and binary solution).

\section{Preliminaries}

In this section, we explain some preliminaries that we use in the rest of our paper. First, in Sect. 4.1, we mention the basic characteristics of DRL and its well-known types such as Q-learning and policy gradient and in Sect. 4.2 LSTM networks are explained.

\subsection{Basic characteristics of the DRL}

In RL algorithm [24], an agent transits from a state to another state until it reaches the final or goal state. The agent receives rewards or punishments for taking actions in its environment. The agent's goal is to maximize its cumulative rewards. These terms can be introduced more formally by Markov decision process tuples $(S, A, R, P, \gamma)$. The first term is " $\mathrm{S}$ " which represents all of the possible states, "A" similarly presents all of the possible action that the agent can perform in its state. " $R$ " is the distribution of possible rewards. " $P$ " sets the transition probability distribution and finally, discount factor $(\gamma)$ determines "how much" the future rewards should be discounted. The reason for using this hyper-parameter is the uncertainties that the agent will face. The agent in the RL algorithm tries to choose actions which provide more rewards. The agent's goal is to reach an optimal policy $(\pi)$. A policy shows which action should be chosen in every state. Simply, a policy maps states to actions and as mentioned, the RL algorithm goal is to learn an optimal policy which maps states to the best possible actions and maximize the expected cumulative discounted reward [24].

$\pi^{*}=\arg \max _{\pi} \mathbb{E}\left[\sum_{t>0} r^{t r_{t} \mid \pi}\right]$ with $s_{0 \sim} p\left(s_{0}\right), a_{t \sim} \pi\left(. \mid s_{t}\right), s_{t+1} p\left(. \mid s_{t}, a_{t}\right)$

In following, Q-learning (a well-known RL type) and deep Q-leaning (DQL) terms and equations will be explained [12]. Two specific Q-learning terms, in which the optimal policy can be defined by these two terms, are Value-function and Q-value function.

Value-function simply shows the goodness of a particular state or more formally it determines the expected cumulative discounted reward that can be received from being in a particular state.

$V^{\pi}(s)=\mathbb{E}\left[\sum_{t \geq 0} r^{t r_{t} \mid s_{0}=s, \pi}\right]$
The Q-value function is slightly more complicated, this time actions are considered too. It shows the expected reward that can be received from being in a particular state, performing a particular action and following the policy afterward.

$Q^{\pi}(s, a)=\mathbb{E}\left[\sum_{t \geq 0} r^{t r_{t} \mid s_{0}=s, a_{0}=a, \pi}\right]$

And, simply optimal Q-value function is the maximum value of the expected reward that can be received from being in a specific state and taking a specific action.

$Q^{*}(s, a)=\max _{\pi} \mathbb{E}\left[\sum_{t \geq 0} r^{t r_{t} \mid s_{0}=s, a_{0}=a, \pi}\right]$

If we know the optimal Q-values for the next time-step, optimal Q-value function satisfies the following Bellman equation:

$Q^{*}(s, a)=\mathbb{E}_{s^{\prime} \sim \varepsilon}\left[r+\gamma \max _{a^{\prime}} Q^{*}\left(s^{\prime}, a^{\prime}\right) \mid s, a\right]$

Finally, the optimal policy $\left(\pi^{*}\right)$ is reachable by taking the optimal action in any state which specified by $Q^{*}$-values.

As it is clear, computing these Q-values for every state action pair $(\mathrm{Q}(\mathrm{s}, \mathrm{a}))$ in a complex problem which has a huge state-space is not feasible. Therefore, neural networks which are one of the best estimators are used in DRL (DQL).

For using neural networks, a network parameter theta $(\theta)$, which represents all of the network's weights must be added and optimal Q-values will be estimated by these weights.

$Q(s, a ; \theta) \approx Q^{*}(s, a)$

Now that a neural network does the approximation part, a loss function should be trained to minimize the bellman equation error. Simply, it shows "how far" are the predicted values from the target values $\left(y_{i}\right)$.

$$
\begin{gathered}
\text { lossfunction : } L_{i}\left(\theta_{i}\right)=\mathbb{E}_{s, a \sim p(.)}\left[\left(y_{i}-Q\left(s, a ; \theta_{i}\right)\right)^{2}\right] \\
\text { where } y_{i}=\mathbb{E}_{s^{\prime} \sim \varepsilon}\left[r+\gamma \max _{a^{\prime}} Q\left(s^{\prime}, a^{\prime} ; \theta_{i-1}\right) \mid s, a\right]
\end{gathered}
$$

Next step is forward passing the loss function and backward passing the gradients (with respect to theta $(\theta)$ ).

$$
\begin{aligned}
\nabla \theta_{i} L_{i}\left(\theta_{i}\right)= & \mathbb{E}_{s, a \sim p(.) ; s^{\prime} \sim \varepsilon}\left[r+\gamma \max _{a^{\prime}} Q\left(s^{\prime}, a^{\prime} ; \theta_{i-1}\right)\right. \\
& \left.-Q\left(s, a ; \theta_{i}\right)\right) \nabla_{\theta_{i}} Q\left(s, a ; \theta_{i}\right)
\end{aligned}
$$

It leads the predicted Q-values close to the target values iteratively.

Another well-known RL approach is policy gradient [25]. In the policy gradient approach, an optimal policy 
will be chosen from a set of policies. In this approach, we need to save some trajectories $(\Gamma)$. Each trajectory contains pieces of information in the form of $\left(s_{0}, a_{0}, r_{0}, s_{1}, ..\right)$. samples are chosen from these trajectories and expected future reward of the chosen samples will be computed, also each policy value will be computed as well. After that, a gradient ascent step should be performed on policy parameters. If the achieved reward from a trajectory $(\Gamma)$ is high, we push up the probability of its consisting actions and vice versa.

This approach usually used for problems that have a complex state-space and computing all of the Q-values are time-consuming. However, the Policy gradient itself suffers from high variance. Therefore, it needs a lot of samples. In the other hand as mentioned, computing Q-values in Q-learning algorithm is challenging. Combining these two approaches called actor-critic approach [26] which we used in our proposed model and will be explained more in Sect. 5.

\subsection{Long short-term memory network}

As mentioned before, neural networks are used in approximation phase in deep RL algorithm. In the MSA problem, we need to memorize some pieces of information due to its sequential and decision-making character. LSTMs [27] which are capable of memorizing long dependencies should be used. In LSTMs we have three main parts (layers) which are shown in Fig. 2.

In the first part, the network decides to omit which pieces of information from the cell. Previous state $\left(\mathrm{h}_{(\mathrm{t}-1)}\right)$ and the current input are the sigmoid function inputs and the sigmoid function (which called forget gate) delete pieces of information that are not important anymore.

$f_{t}=\sigma\left(W_{f} \cdot\left[h_{t-1}, x_{t}\right]+b_{f}\right)$

where $f_{t}$ is the forget gate's activation vector and $W_{f}$ represents the weight matrices and $b_{f}$ is the forget gate's bias

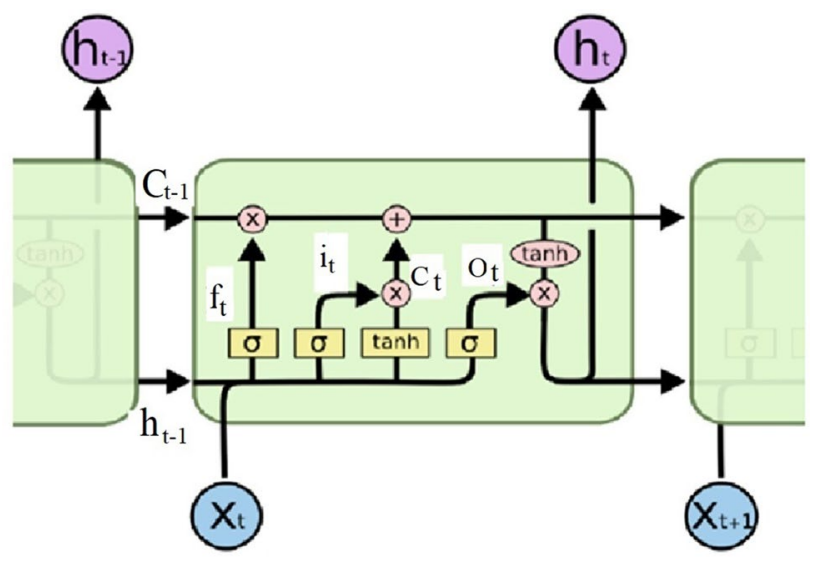

Fig. 2 LSTM network structure (layers) [38] vector $\left(\mathrm{i}_{\mathrm{t}}, \mathrm{W}_{\mathrm{i}}, \mathrm{b}_{\mathrm{i}}, \mathrm{o}_{\mathrm{t}}, \mathrm{W}_{\mathrm{o}}, \mathrm{b}_{\mathrm{o}}\right.$ defined similarly for the input gate and the output gate) [27].

The second part consists of a sigmoid and a tanh function (which called input gate). The Sigmoid function in the second part decides which values to let through ( 0 or 1 ) and the tanh assigns weights to the chosen values according to their level of importance.

$i_{t}=\sigma\left(W_{i} \cdot\left[h_{t-1}, x_{t}\right]+b_{i}\right)$

$C_{t}^{\sim}=\tanh \left(W_{C} \cdot\left[h_{t-1}, x_{t}\right]+b_{C}\right.$

where $C_{t}$ is the cell state vector [27].

Finally, in the third part, which also consists of a sigmoid and tanh function, the network's output will be decided. The sigmoid function in the third layer determines what parts of the cell state make it to the output and the tanh pushes the cell state values between 1 and -1 . In the end, multiplying these values with the output of the sigmoid function determines the network's final output.

$o_{t}=\sigma\left(W_{o}\left[h_{t-1}, x_{t}\right]+b_{o}\right)$

$h_{t}=o_{t} * \tanh \left(C_{t}\right)$

where $h_{t}$ is the output vector of the LSTM neuron [27].

\section{Our proposed approach}

In this section, we introduce our deep reinforcement learning model. Finding an order or simply a permutation of $n$ number of sequences in which we have the maximum rewards (SP score), is the goal of the MSA problem in our model.

At first, the Markov decision process term should be defined for the MSA problem. " $\mathrm{S}$ " which represent all of the possible states is consists of $\frac{n^{n+1}-1}{n-1}$ states for $n$ number of sequences. " $\mathrm{A}$ " is consists of $n$ actions for $n$ number of sequences. Reward function should be defined based on the SP score and this fact that the chosen actions must be distinct. Therefore, the agent receives a big negative value $(-\infty)$ if the agent's chosen action is not distinct. For the first step in which there is no other sequence to compare with $\left(r\left(n_{k} \mid n_{k-1}\right)\right.$ if $\left.k=1\right)$ the reward is 0 . And, for the rest of the occurrences, we used SP score (match, mismatch, and gap score) value based on the reward and penalty values which introduced in Sect. 2. Our discount factor set to 0.95 and epsilon value was 1.0 and it decayed through the process because we wanted to explore more in early episodes.

The alignment process was progressive alignment method based and works in the way that shown in Fig. 3. Sequence 1 aligned with sequence 2 and aligned sequence of the mentioned sequences aligned with sequence 3 and so on. 


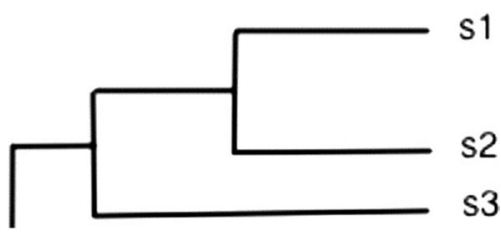

Fig. 3 Alignment order in the progressive alignment method

\begin{tabular}{|c|c|c|c|c|c|c|c|c|c|c|c|c|c|c|}
\hline & - & $\bar{A}$ & $\mathrm{G}$ & G & $\mathrm{C}$ & $\mathrm{T}$ & $\bar{A}$ & $\mathrm{~T}$ & $\mathrm{C}$ & $\mathrm{A}$ & $\mathrm{C}$ & $\bar{C}$ & $\mathrm{~T}$ & $\mathrm{G}$ \\
\hline & $\mathrm{T}$ & A & G & - & C & $\mathrm{T}$ & A & C & C & A & - & - & - & G \\
\hline & C & A & G & - & C & $\mathrm{T}$ & A & C & C & A & - & - & - & $G$ \\
\hline & C & A & G & - & C & $\mathrm{T}$ & A & $\mathrm{T}$ & C & A & C & - & G & G \\
\hline & C & A & G & - & C & $\mathrm{T}$ & A & $\mathrm{T}$ & C & G & C & - & G & G \\
\hline A & & 1 & & & & & 1 & & & .8 & & & & \\
\hline C & .6 & & & & 1 & & & .4 & 1 & & .6 & .2 & & \\
\hline G & & & 1 & .2 & & & & & & .2 & & & .4 & 1 \\
\hline $\mathrm{T}$ & .2 & & & & & 1 & & .6 & & & & & .2 & \\
\hline - & .2 & & & .8 & & & & & & & .4 & .8 & .4 & \\
\hline
\end{tabular}

Fig. 4 A simple profile [39]

Pairwise alignment is a really easy task in dynamic programming algorithm but for multiple alignments, it is a challenging and time-consuming task. For example, in aligning a sequence with $n-1$ number of aligned sequences, we have a character in a column that matches against all of the other aligned sequences in that particular column. Therefore, alignments should be performing between a sequence and a profile [8]. A profile of prealigned sequences shown in Fig. 4. Simply a profile contains the frequency of each character's occurrences.

Having profiles of the pre-aligned sequences enables us to use an extension of Needleman-Wunsch method [28] which proposed for finding an optimal alignment between two sequences.

As mentioned earlier, we used LSTM networks for approximating Q-values. Simply LSTMs remember events which happened in the hidden layers and hidden layers feedback those important events into the next layers. In our proposed approach we used three LSTMs as hidden layers also, after each LSTM we put a dropout layer in which prevents the network from over-training. Simply, a dropout layer deactivates some of the hidden layer's neurons. For our model, we set dropout range to 0.3 which means 30 percent of the units (neurons) won't work in every episode randomly. Each LSTM network in our model had 40 neurons and the inputs of the network were the state's information which is needed for computing SP score. A list of so far chosen sequences are also crucial (actions must be distinct) and we selected a dense (fully-connected) layer in size of $n$ (number of actions (sequences)) for our output layer (Fig. 5).

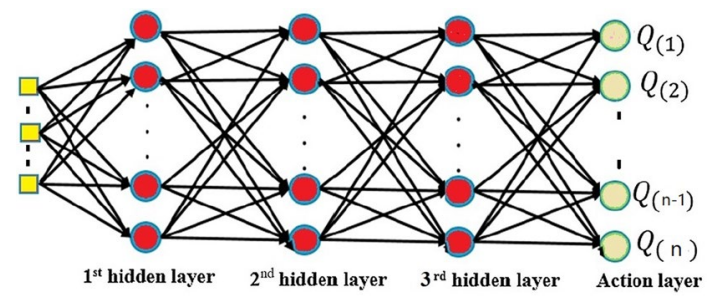

Fig. 5 Our deep Q-Network structure

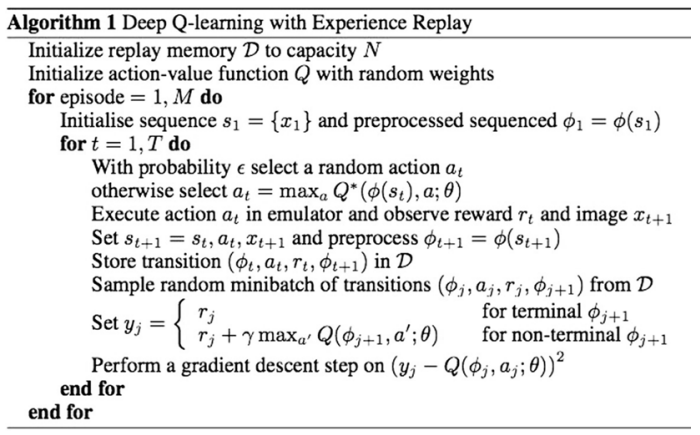

Fig. 6 Pseudo-code of DQN with experience-replay method [12]

A solution to the MSA problem is a sequence of distinct actions $\left(a_{1}, a_{2}, \ldots, a_{n}\right)$ in which each action represents the position of each sequence (a valid permutation).

We used a non-linear approximator (neural network). This usage may affect the process (diverged or unstable). Correlation between observed samples and changes in the policy due to the small changes (updates) of the Q-values are the main reasons for the instability. Also, learning from batches of consecutive samples is not useful. For example, putting a particular sequence in a specific position which is not its optimal position and changing the positions of other sequences won't provide any efficient result. For preventing these problems, we used the experience-replay method [29]. In the experience-replay method, we use saved transitions memories which contains pieces of information such as state, action, reward, and next-state. Then mini-batch sampling step and also, a gradient descent step should be performed. A pseudo-code of deep Q-learning with the usage of the experience-replay method shown in Fig. 6.

As it is shown in the mentioned pseudo-code first, we start off with initializing our replay memory to the capacity of $\mathrm{N}$. Then we initialize our Q-network with random weights after processing $\mathrm{M}$ episode (this is our training episodes) then initialize our state and for each time step, we select a random action with a small probability (it is crucial to have 
sufficient exploration because we want to sample different parts of the state-space) otherwise, we try to select from the greedy action from the current policy (most of the time we take the greedy action that we think is a good policy of the type of actions that we want to take and sates that we want to visit and with a small probability we sample something random). After taking the selected action $\left(a_{t}\right)$, we observe the next reward and the next state $\left(r_{t}, s_{t+1}\right)$. Then, we store this transition in our replay memory that we are building up. After these steps, we train our network by the experiencereplay method. For performing this process, a sample of a

\section{Discussion and results}

Compared to the supervised approaches that were proposed for the MSA problem, in RL approach we need no external supervision and the solution is learned from the achieved rewards through the training phase.

The time complexity of our proposed approach is not exponential. To set the time complexity of our approach at first, we should define neural networks complexity. the time complexity of an LSTM network expressed as:

$\mathrm{W}_{\mathrm{LSTM}}=\mathrm{num} \_\mathrm{c} * \mathrm{num} \_\mathrm{c} * 4+\mathrm{num} \_\mathrm{i} * \mathrm{num} \_\mathrm{c} * 4+$ num_c $*$ num_o + num_c $* 3$

random mini-batches of transitions that we stored earlier from the replay memory is taken. At last, we perform a gradient descent step. This is our full training loop and by continuously performing these steps, sampling mini-batches and using the experience-replay method our network's weights will be updated.

As mentioned in the first section, we also, used the actorcritic algorithm for faster convergence. The actor-critic method is the result of combining Q-learning and policy gradients approaches. After training both the critic (the Q-function) and the actor (the policy), when the actor wants to choose an action, the critic informs the actor if its chosen action is the best choice or not.

Over-fitting due to the ability of the deep learning models to adapt freely to large amount of complex data is very common. We had issues in noisy datasets and the model showed high variance. To address this issue which is improving the generalization of our neural network we used the early stopping method. In the mentioned method, we divide datasets into sub-parts. First part is responsible for computing the gradient and updating the network's weights and biases. We monitor the second part (which is called the validation set) errors during the training stage. By these pieces of information, we can easily decide to stop or continue the training process. Usually in the early stage of the training process validation error decreases and when the network starts to overfit the data, the error also starts to rise and when we reach this point in a specific number of iteration, the training process will be stopped and the best values of the weights and biases will be returned.

Other important implication details are: the size of the mini-batches was 32. "Adam" optimizer which is the substitution algorithm for gradient descent in deep learning models was used. where num_i is the number of network's inputs, num_c is the number of memory cells and num_o is the number of outputs. The computational complexity of learning LSTM models per weight and time step with stochastic gradient descent optimization technique is $O$ (1). Therefore, the learning process time complexity per time-step is $\mathrm{O}(\mathrm{W})$ [30]. The time complexity of the $\mathrm{RL}$ approach is $\theta(n . k)$, where $n$ is the number of sequences and $k$ is the number of episodes. Also, in the DRL approach with the experience-replay method, we should add $\theta$ (n. logn) for inserting and $\theta(n)$ for sampling, which could get reduced by using a data structure called sumtree instead of using arrays. Sumtree is a binary tree which each node has two leaves at most. Updating tree and sampling have time complexity of $\theta(\log n)$ with sumtree structure.

Comparing our approach with other approaches provided the following results: As mentioned in Sect. 3, many genetic-based approaches proposed for the MSA problem. A comparison between our approach and two of the best genetic-based algorithms [3] and [31] on the Papio Anubis dataset [32] and Hepatitis C virus dataset [32] shown in Fig. 7. Papio Anubis dataset contains 5 DNA sequences and the average length of its sequences is 1093 and Hepatitis $C$ virus dataset contains 10 DNA sequences and the average length of its sequences is 212 , both of them are part of EMBL dataset. Due to the more efficiency of the DRL
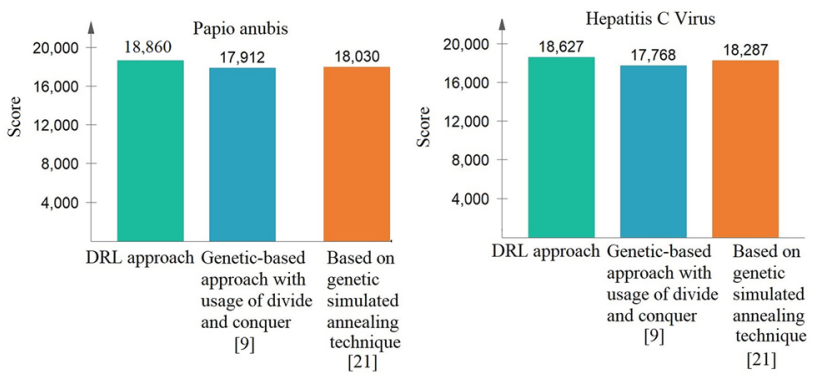

Fig. 7 A comparison between our approach and genetic-based approaches 
approach, our approach achieved more score than the mentioned approaches.

Another comparison between our approach and two of the best-known tools which are ClustalW and MAFFT made on lemur, gorilla and mouse (LGM) dataset [33] and Rat, Lemur and opossum (RLO) dataset [33]. LGM dataset contains mentioned animals DNA sequences and the average size of its sequences is 93 and RLO dataset contains the third exon sequences of the beta-globin gene of the mentioned animal and the average size of its sequences is 129. Both of them are DNA datasets.

As shown in Fig. 8 our approach has an equal achieved score on lemur, gorilla and mouse dataset (The maximum score could ever achieve from LGM dataset is 345). However, our approach has more CS (column score), AL (alignment length) and more EM (exact match) values which is shown in Fig. 9.

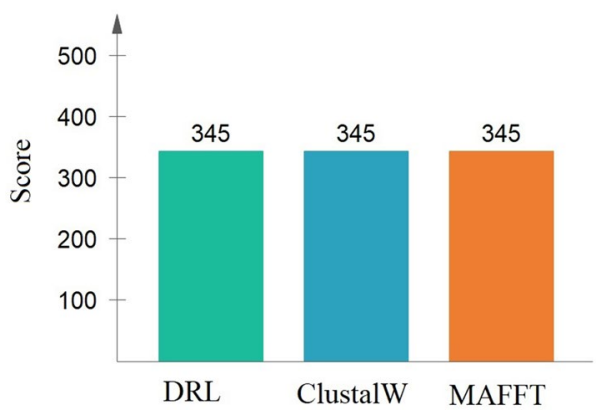

Fig. 8 Comparison between DRL, ClustalW, and MAFFT on LGM data set

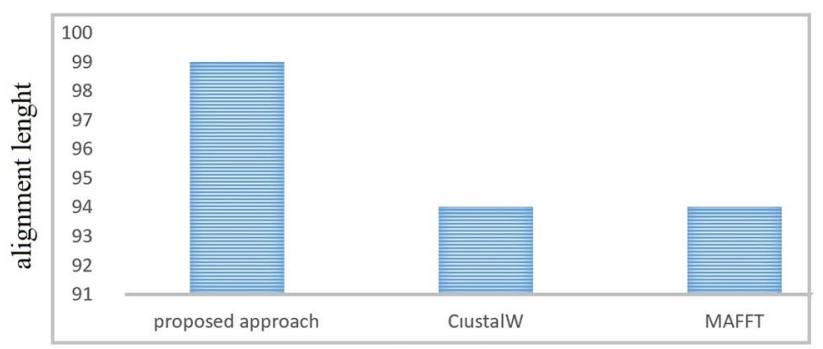

approaches (tools)
Our approach outperformed the other two tools on Rat, Lemur and opossum dataset as shown in Fig. 10.

Continue with comparing our approach results with state-of-art approaches which proposed for the MSA problem, we reach to the Xiang and et al. [33] approach which utilized Ant colony with genetic algorithm based on planar graph on Rat, Lemur and opossum dataset. The result is shown in Fig. 11.

After comparing with older well-known approaches. In this point of the results section, we compare our approach results with newer approaches especially RLbased approaches $[8,9]$. We test our results with another RL-based approach [8] on LGM, RLO, Papio Anubis, Hepatitis $C$ Virus datasets and two other datasets which are dataset 469 from oxbench_mdsa_all [34] and dataset 429

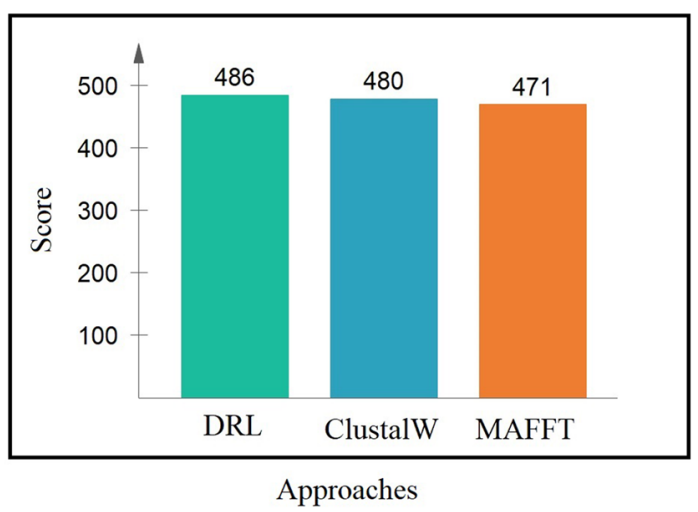

Fig. 10 Comparison between our approach, ClustalW, and MAFFT on RLO data set

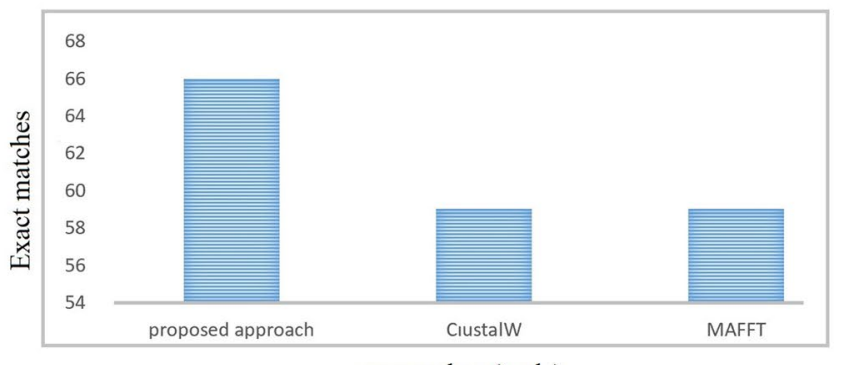

approaches (tools)

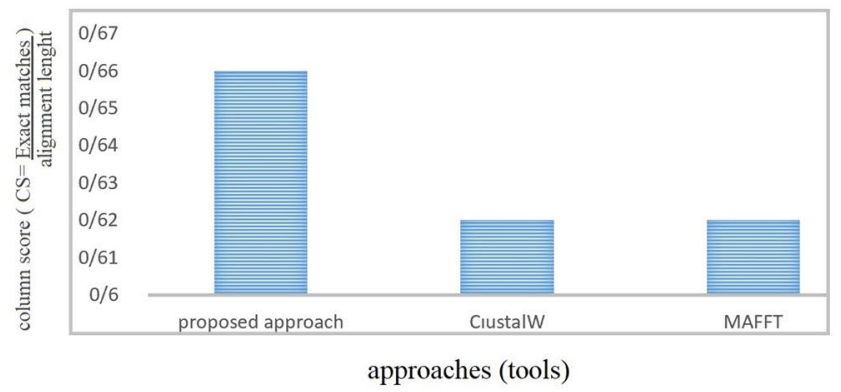

Fig. 9 AL, EM, CS value comparison on LGM data set 


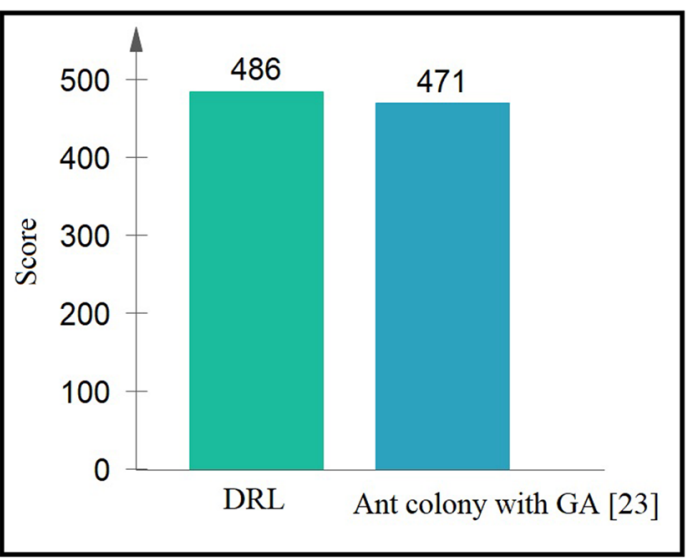

Approaches

Fig. 11 Comparison between our approach and Ant colony with the genetic algorithm on RLO data set

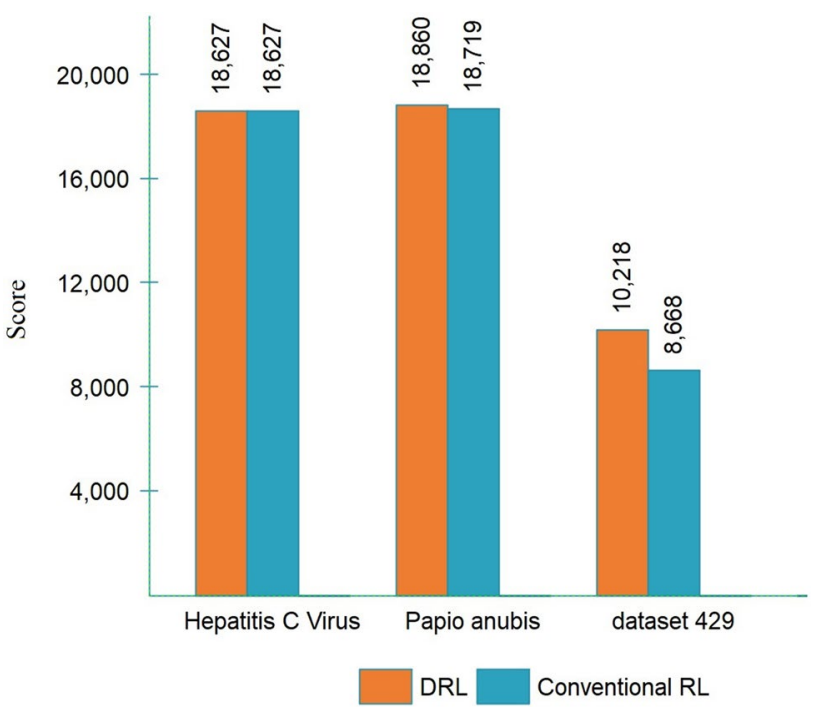

Fig. 12 Comparison between DRL and Conventional RL (part 1)

from oxbench_mdsa_all [34]. Dataset 469 contains 3 DNA sequences and the average size of its sequences is 332 and dataset 429 contains 12 DNA sequences and the average length of these sequences is 171. Due to the score value difference between these datasets (Papio Anubis $=18,627$ and LGM $=345$ ), we divide these 6 datasets into two part for better visibility. The first part of the datasets includes Hepatitis C Virus dataset, Papio anubis dataset and dataset 429. The result is shown in Fig. 12.

And the second part of the datasets contains LGM dataset, RLO dataset, and dataset 469 and the result is shown in Fig. 13.

As it is clearly shown in Figs. 12 and 13, conventional RL has an acceptable performance in small datasets (has small

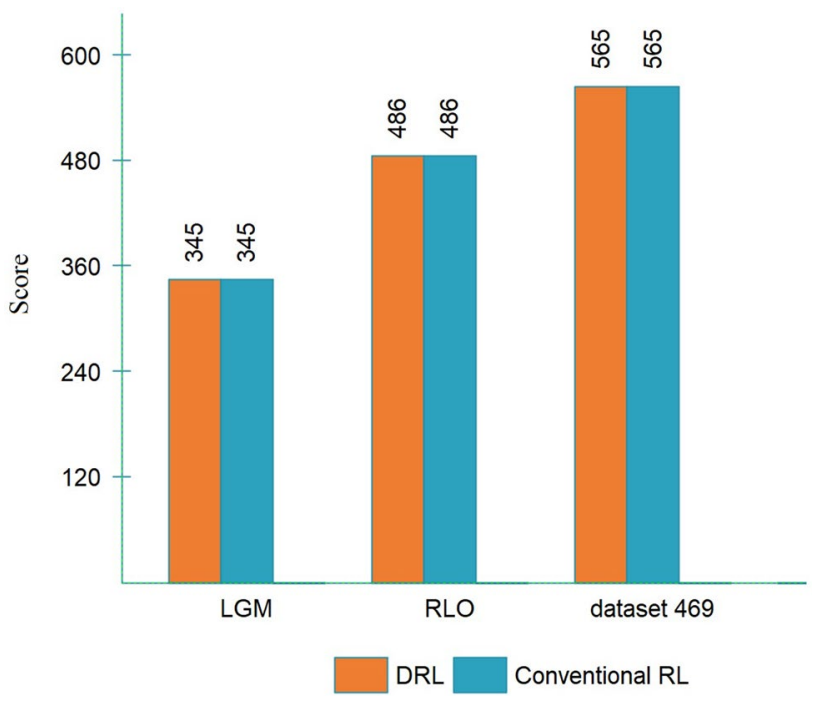

Fig. 13 Comparison between DRL and Conventional RL (part 2)

score value) however, as we tested more complex datasets which require more states in $\mathrm{RL}$ algorithm, DRL approach outperforms traditional RL approach. The reason for this performance gap is the efficiency difference between the neural network structure and q-tables. Computing all the $\mathrm{Q}(\mathrm{s}, \mathrm{a})$ values and memorizing them in table structure in a complex problem which has a complex environment and states is very time-consuming. However, the convergence process can be reached much faster by using neural network structures such as LSTMs and methods such as actorcritic. The following results supports this claim.

After comparing the approaches from Scoring point of view, now we compare our approach with another RLbased approach [9] (this approach is more efficient than [8] approach and provided its running-time) in case of computational time length. For this comparison, two real-life datasets which are collected from Babeș-Bolyai research institute [35] were used. The datasets contain mitochondrial human DNA sequences. The first dataset consists of 8 sequences with the average length of 365 and the second dataset consists of 6 sequences and the average length of its sequences is 362 . In [9], they used a different gap penalty, match and mismatch values and we changed our

Table 1 A runtime, score and column comparison between our approach and another RL-based approach

\begin{tabular}{llll}
\hline Datasets & Score & Column score & Time (s) \\
\hline Dataset 1 & DRL: 36664 & DRL: 0.842 & DRL: 486 \\
& RL: 36645 & RL: 0.839 & RL: 1334 \\
Dataset 2 & DRL: 20337 & DRL:0.911 & DRL:35 \\
& RL: 20337 & RL: 0.911 & RL: 101 \\
\hline
\end{tabular}


Table 2 A detailed comparison with other alignment's factors

\begin{tabular}{|c|c|c|c|c|c|c|c|c|c|c|c|c|c|c|c|c|}
\hline \multirow[t]{2}{*}{ Datasets } & \multicolumn{4}{|c|}{ Our approach } & \multicolumn{4}{|l|}{ MAFFT } & \multicolumn{4}{|c|}{ RL-based approach [8] } & \multicolumn{4}{|c|}{ ClustalW } \\
\hline & Score & EM & $\mathrm{AL}$ & CS & Score & EM & $\mathrm{AL}$ & CS & Score & EM & $A L$ & CS & Score & EM & $A L$ & CS \\
\hline RLO dataset & 486 & 87 & 138 & 0.65 & 471 & 81 & 129 & 0.62 & 486 & 87 & 133 & 0.65 & 480 & 83 & 130 & 0.63 \\
\hline Papio anubis & 18,860 & 918 & 1110 & 0.82 & 18,860 & 907 & 1098 & 0.82 & 18,719 & 918 & 1110 & 0.82 & 18,827 & 905 & 1098 & 0.82 \\
\hline Hepatitis C virus & 18,627 & 198 & 212 & 0.93 & 18,627 & 198 & 212 & 0.93 & 18,627 & 198 & 212 & 0.93 & 18,627 & 198 & 212 & 0.93 \\
\hline LGM dataset & 345 & 66 & 99 & 0.66 & 345 & 59 & 94 & 0.62 & 345 & 66 & 99 & 0.66 & 345 & 59 & 94 & 0.62 \\
\hline Dataset 429 & 10,218 & 43 & 206 & 0.20 & 10,218 & 35 & 183 & 0.19 & 8668 & 43 & 206 & 0.20 & 9575 & 35 & 186 & 0.18 \\
\hline Dataset 469 & 565 & 171 & 378 & 0.45 & 549 & 122 & 342 & 0.35 & 565 & 171 & 378 & 0.45 & 464 & 134 & 359 & 0.37 \\
\hline
\end{tabular}

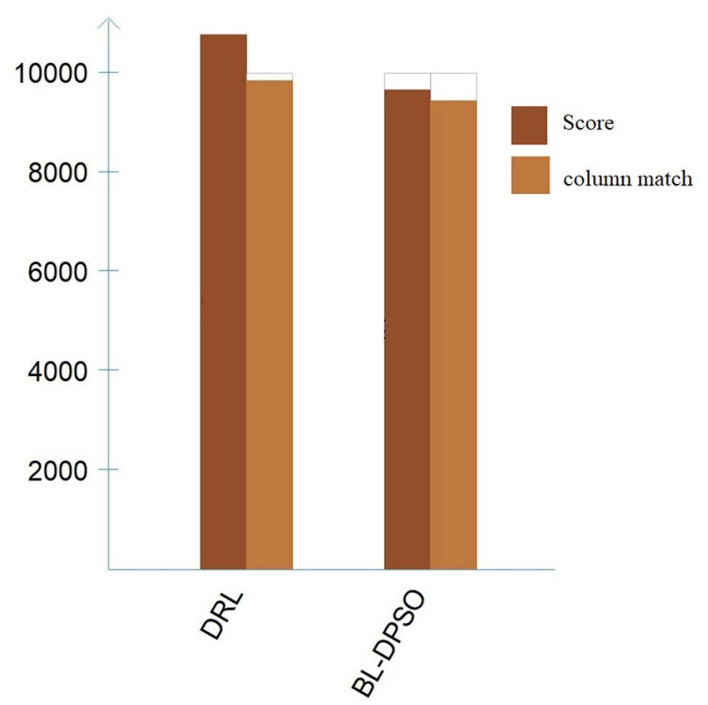

Fig. 14 Comparison between DRL and BL-DPSO

values to their chosen values to make this comparison (the linear gap penalty is selected). Clearly Table 1 . Shows the faster convergence process of the DRL approach. in more complex datasets the difference shows itself better.

We compared our approach with two best well-known tools and another RL-based approach on other datasets with more details it is shown in Table 2.

We conclude this section by comparing our approach with the most recent paper in solving MSA problem (time of writing our paper). Efficient Bi-level discrete PSO variant (BL-DPSO) [16] as said before in Sect. 3, it is consisting of two level. Level one, processes on optimizing dimension for entire swarm and the second level works on optimizing each and every particles position. We compared our approach with BL-DPSO approach on K7-long BRAlibase dataset [36] which is an RNA dataset. The result is shown in Fig. 14.

As shown in Fig. 14 our approach outperformed on BRAlisbase dataset. Using neural network really affects the result in heavy and complex datasets such as BRAlibase dataset and other reason is RL based approaches really outperforms PSO based approaches in decision-making problems with complex environment such as protein folding problem, genomic sequence analysis and gene-finding [37].

\section{Conclusion and future development}

In this paper, we proposed a deep reinforcement learning approach for finding an optimal solution in the MSA problem. The MSA problem is one of the most important and fundamental problems in bioinformatics. In the present study, the MSA problem modeled as an RL problem then DRL approaches such as deep Q-learning and policy gradient method proposed for this problem and by combining them, a critic-actor method also applied to the MSA problem. For approximating Q-function values we used LSTMs because of their ability to memorizing events. In the training phase, we used the experience-replay method for avoiding correlation between samples and better efficiency. Our approach shows outstanding results in wellknown DNA datasets in much less time than other RL approaches.

Future development can be addressed by using other structures for the network, using more optimized local search methods, different network and model parameters' values such as discount factor, number of LSTMs'layers and units. Also, different reward function based on other comparable values could be useful.

\section{Compliance with ethical standards}

Conflict of interest The authors declare that they have no conflict of interest.

Human and animal rights This article does not contain any studies with human participants or animals performed by any of the authors. 


\section{References}

1. Eger S (2013) Sequence alignment with arbitrary steps and further generalizations, with applications to alignments in linguistics. Inf Sci 237:287-304. https://doi.org/10.1016/j. ins.2013.02.031

2. Wang L, Jiang $T$ (1994) On the complexity of multiple sequence alignment. Comput Biol 4:337-348. https://doi.org/10.1089/ cmb.1994.1.337

3. Chen SM, Lin CH (2005) Multiple DNA sequence alignment based on genetic algorithms and divide-and-conquer techniques. Int J Appl Sci Eng 3:89-100

4. Agarwal $P$, Chauhan $R$ (2013) Alignment of multiple sequences using GA method. Int J Emerg Technol Comput Appl Sci (IJETCAS) 13-177:412-421

5. Katoh K, Misawa K, Kuma K, Miyata T (2002) MAFFT: a novel method for rapid multiple sequence alignment based on fast Fourier transform. Nucleic Acids Res 30:3059-3066. https://doi. org/10.1093/nar/gkf436

6. Rasmussen TK, Krink T (2003) Improved hidden markov model training for multiple sequence alignment by a particle swarm optimization-evolutionary algorithm hybrid. BioSystems 72:517. https://doi.org/10.1016/s0303-2647(03)00131-x

7. Nasser S, Vert G, Nicolescu M, Murray A (2007) Multiple sequence alignment using fuzzy logic. In: 2007 IEEE symposium on computational intelligence and bioinformatics and computational biology, Honolulu, HI, 2007, pp 304-311. https://doi. org/10.1109/cibcb.2007.4221237

8. Mircea IG, Bocicor I, Czibula G (2018) A reinforcement learning based approach to multiple sequence alignment. Soft computing applications. SOFA 2016. Advances in intelligent systems and computing, vol 634. Springer, Cham. https://doi. org/10.1007/978-3-319-62524-9_6

9. Mircea I, Bocicor M, Dıncu A (2014) On reinforcement learning based multiple sequence alignment. Studia Universitatis "Babes-Bolyai", Informatica LIX, 50- 65. Retrieved from https:// pdfs.semanticscholar.org

10. Larkin M, Blackshields G, Brown N, Chenna R, McGettigan P, McWilliam H, Valentin F, Wallace I, Wilm A, Lopez R, Thompson J, Gibson T, Higgins D (2007) ClustalW and clustalX version 2.0. Bioinformatics 23(21):2947-2948. https://doi.org/10.1093/bioin formatics/btm404

11. Katoh S (2013) MAFFT multiple sequence alignment software version 7: improvements in performance and usability. Mol Biol Evol 30:772-780. https://doi.org/10.1093/molbev/mst010

12. Mnih V, Kavukcuoglu K, Silver D, Graves A, Antonoglou I, Wierstra $D$, et al (2013) Playing Atari with deep reinforcement learning. Technical report Deepmind Technologies. Retrieved from https ://arxiv.org/abs/1312.5602

13. Lipman D, Altschul S, Kececioglu J (1989) A tool for multiple sequence alignment. Proc Natl Acad Sci USA 86:4412-4415. https://doi.org/10.1073/pnas.86.12.4412

14. Chen Y, Pan Y, Chen L, Chen J (2006) Partitioned optimization algorithms for multiple sequence alignment. In: Proceedings of the 20th international conference on advanced information networking and applications, pp 618-622. https://doi.org/10.1109/ aina.2006.260

15. Rubio-Largo A, Vega-Rodríguez M, González-Álvarez D (2016) Hybrid multiobjective artificial bee colony for multiple sequence alignment. Appl Soft Comput 41:157-168. https:// doi.org/10.1016/j.asoc.2015.12.034

16. Lalwani S, Sharma H, Krishna M, Kusum D (2019) An efficient bi-level discrete PSO variant for multiple sequence alignment: theory and applications. ICHSA 2018. https://doi. org/10.1007/978-981-13-0761-4_76
17. Chenna R, Sugawara H, Koike T, Lopez R, Gibson T, Higgins D, Thompson J (2003) Multiple sequence alignment with the clustal series of programs. Nucleic Acids Res 31(13):3497-3500. https://doi.org/10.1093/nar/gkg500

18. Katoh K, Frith M (2012) adding unaligned sequences into an existing alignment using MAFFT and LAST. Bioinformatics 28(23):3144-3146. https://doi.org/10.1093/bioinformatics/bts57 8

19. Radhouene Aniba M, Poch O, Marchler-Bauer A, Dawn Thompson J (2010) AlexSys: a knowledge-based expert system for multiple sequence alignment construction and analysis. Nucleic Acids Res 38(19):6338-6349. https://doi.org/10.1093/nar/gkq52 6

20. Tavallali P, Yazdi M, Khosravi MR (2019) Robust cascaded skin detector based on AdaBoost. Multimedia Tools Appl 78(2):2599_ 2620. https://doi.org/10.1007/s11042-018-6385-7

21. Tavallali P, Yazdi M (2015) Robust skin detector based on AdaBoost and statistical luminance features. In: Proceedings of IEEE ICTCK-2015, Mashhad. https://doi.org/10.1109/ICTCK .2015 .7582653

22. Breiman L (1984) Classification and regression trees. Routledge, New York. https://doi.org/10.1201/9781315139470

23. Criminisi A, Shotton J, Konukoglu E (2012) Decision forests: a unified framework for classification, regression, density estimation, manifold learning and semi-supervised learning. Now Publishers Inc, Hanover. https://doi.org/10.1561/0600000035

24. Sutton RS, Barto AG (1998) Reinforcement learning: an introduction. MIT Press, Cambridge. https://doi.org/10.1109/ tnn.1998.712192

25. Sutton RS, McAllester DA, Singh SP, Mansour Y (1999) Policy gradient methods for reinforcement learning with function approximation. Adv Neural Inf Process Syst (NIPS) 12:1057-1063

26. Konda V. R, Tsitsiklis J (2000) Actor-critic algorithms. In: Proceedings of the 1999 conference advances in neural in-formation processing systems vol 12, pp 1008-1014, (Denver, Colorado, 2000). https://doi.org/10.1137/S0363012901385691

27. Hochreiter S, Schmidhuber J (1997) Long short-term memory. Neural Comput 9:1735-1780. https://doi.org/10.1162/ neco.1997.9.8.1735

28. Needleman S, Wunsch C (1970) A general method applicable to the search for similarities in the amino acid sequence of two proteins. J Mol Biol 48(3):443-453. https://doi.org/10.1016/00222836(70)90057-4

29. Schaul T, Quan J, Antonoglou D (2015) Prioritized experience replay. arXiv preprint arXiv:1511.05952. https://arxiv.org/ abs/1511.05952

30. Sak H, Senior A, Beaufays F (2014) Long short-term memory based recurrent neural network architectures for large vocabulary speech recognition. ArXiv e prints. Retrieved from https:// arxiv.org/abs/1402.1128

31. Chen SM, Lin CH (2007) Multiple DNA sequence alignment based on genetic simulated annealing techniques. Inf Manag Sci 18:97-111

32. Kanz C, Aldebert $P$, Althorpe $\mathrm{N}$ et al (2005) The EMBL nucleotide sequence database. Nucleic Acids Res 36:D29-D33. https://doi. org/10.1093/nar/gki098

33. Xiang X, Zhang D, Qin J, Yuanyuan F (2010) Ant colony with genetic algorithm based on planar graph for multiple sequence alignment. Inf Technol J 9(2):274-281. https://doi.org/10.3923/ itj.2010.274.281

34. Carroll H, Beckstead W, O'Connor T, Ebbert M, Clement M, Snell Q, McClellan D (2007) DNA reference alignment benchmarks based on teritary structure of encoded proteins. Bioinformatics 23(19):2648-2649. https://doi.org/10.1093/bioinformatics/ btm389 
35. Institute of Interdisciplinary Research in Bio-Nano-Sciences. http://bionanosci.institute.ubbcluj.ro/

36. Wilm A, Mainz I, Steger G (2006) An enhanced RNA alignment benchmark for sequence alignment programs. Algorithms Mol Biol 1:19. https://doi.org/10.1186/1748-7188-1-19

37. Min S, Lee B, Yoon S (2016) Deep learning in bioinformatics. Brief Bioinform 18(5):851-869. https://doi.org/10.1093/bib/bbw068

38. https://colah.github.io/posts/2015-08-Understanding-LSTMs. Accessed 2 Feb 2019
39. https://www.ebi.ac.uk/training/online/course/introductionprotein-classification-ebi/what-are-protein-signatures/signa ture-types/what-are-. Accessed 2 Feb 2019

Publisher's Note Springer Nature remains neutral with regard to jurisdictional claims in published maps and institutional affiliations. 